\title{
Synchronization of delay-coupled gyrotron oscillators
}

\author{
A.B. Adilova ${ }^{1}$, S.A. Gerasimova ${ }^{1}$, N.M. Ryskin ${ }^{1,2}$ \\ ${ }^{1}$ Saratov State University, Saratov, Russia, usachevasa@gmail.com \\ ${ }^{2}$ Saratov Branch, Institute of Radio Engineering and Electronics, Saratov, Russia
}

\section{Introduction}

The most important application of high-power continuous-wave (CW) gyrotrons is electron cyclotron plasma heating for nuclear fusion [1]. For such application, a large number of gyrotrons should be used to obtain a very high power. For example, in ITER it is planned to use 26 gyrotrons with $1 \mathrm{MW}$ power level. Providing coherence of radiation of large number of high-power microwave sources is a challenging task. Among the possible solutions there are either injection locking by external master oscillator with stabilized frequency $[2,3]$, or mutual synchronization of two or more oscillators [4].

In this paper, a model of two delay-coupled gyrotron oscillators is developed and basic regimes of the mutual interaction are studied. Time delay of the coupling signal traveling between two gyrotrons strongly affects the pattern of phase-locking regimes.

\section{Model}

In [5], we developed a basic theory of mutual phase locking of two limit-cycle oscillators coupled with delay. The delay in coupling provides essential features of the pattern of synchronization. In particular, it is very sensitive to the phase shift of the coupling signal propagating between two counterparts. However, the analysis presented in [5] is valid only in the case of weak coupling and small frequency mismatch. In this paper, we consider a more general model of two coupled gyrotron oscillators, which are assumed identical except a small frequency mismatch. The model is described by two delaydifferential equations (DDE)

$$
\begin{aligned}
& \frac{d A_{1}}{d t}+i \frac{\Delta}{2} A_{1}+A_{1}=I_{s} \Phi\left(A_{1}\right) \cdot A_{1}+\rho e^{-i \psi} A_{2}(t-\tau), \\
& \frac{d A_{2}}{d t}-i \frac{\Delta}{2} A_{2}+A_{2}=I_{s} \Phi\left(A_{2}\right) \cdot A_{2}+\rho e^{-i \psi} A_{1}(t-\tau) .
\end{aligned}
$$

In (1), $A_{1,2}$ are slow amplitudes, $\Delta$ is normalized frequency mismatch, $\Phi=\Phi^{\prime}+i \Phi^{\prime \prime}$ is the complex gain factor, $I_{s}$ is the normalized beam current parameter, $\rho$ is the coupling strength, $\tau=\omega_{0} t_{d} / 2 Q$ is the normalized delay time $t_{d}$, and $\psi=\omega_{0} t_{d}$.

In the quasi-linear theory [6,7], the complex gain factor is obtained as a polynomial expansion $\Phi=\alpha-\beta|A|^{2}-\gamma|A|^{4}-\ldots$ as a result of approximate solution of the electron motion equations. However, this approximation is valid only at a slight excess of the startoscillation current and, consequently at low efficiency. Thus, to extend the range of validity of the model, we obtain $\Phi$ by fitting the numerical solution of the equations of time-domain gyrotron theory with fixed Gaussian profile of the RF field [7]. First, oscillation amplitude $A$ and frequency $\Omega$ are calculated for an uncoupled gyrotron at different $I_{s}$. In a steady-state mode, $\Phi, A$ and $I_{s}$ satisfy the relations

$$
1=I_{s} \Phi^{\prime}(A), \Omega=I_{s} \Phi^{\prime \prime}(A)=\Phi^{\prime \prime}(A) / \Phi^{\prime}(A) .
$$

From (2), one can evaluate $\Phi^{\prime}$ and $\Phi^{\prime \prime}$.
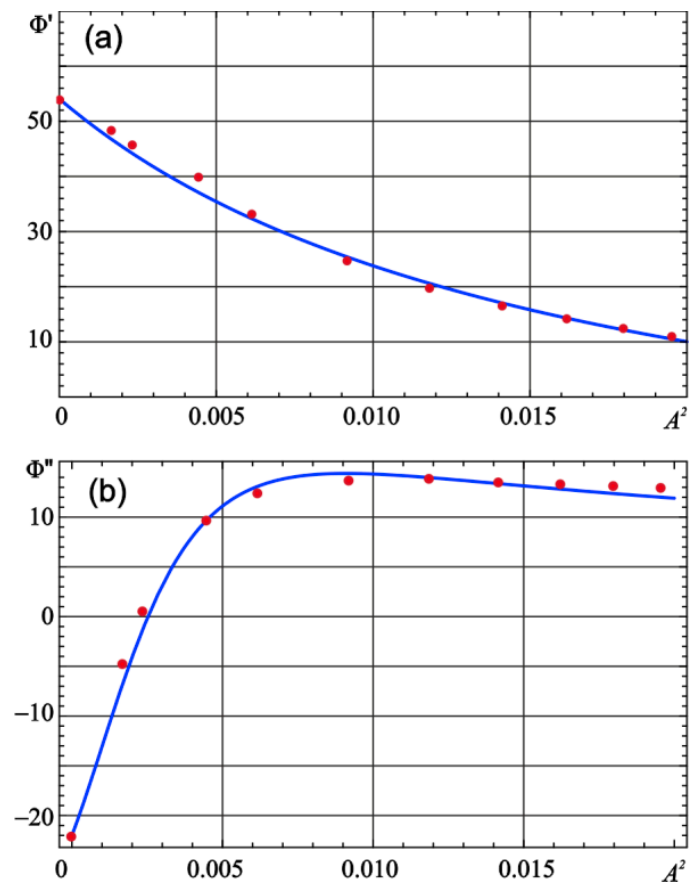

Fig. 1. The real (a) and imaginary (b) parts of the complex gain factor $\Phi$ for gyrotron in soft excitation mode

Consider a gyrotron with the following parameters: cyclotron resonance mismatch $\Delta_{H}=0.4$, and normalized cavity length $\mu=10$. In that case, the gyrotron operates in the soft self-excitation mode. In Fig. 1, the results of numerical simulations shown with circles are compared with fit by formulas

$$
\begin{gathered}
\Phi^{\prime}\left(|A|^{2}\right)=\frac{\alpha^{\prime}-\beta^{\prime}|A|^{2}}{1+\delta^{\prime}|A|^{2}}, \\
\Phi^{\prime \prime}\left(|A|^{2}\right)=\frac{\alpha^{\prime \prime}+\beta^{\prime \prime}|A|^{2}+\gamma^{\prime \prime}|A|^{4}}{1+\delta^{\prime \prime}|A|^{4}},
\end{gathered}
$$

which provide fairly accurate fitting in a wide range of the amplitudes.

\section{Results}

Numerical simulation in a wide range of parameters confirms the basic pattern of synchronization revealed in [5]. In Fig. 3(a), domains of synchronization on the $(\Delta, \rho)$ plane are presented for $I_{s}=0.05, \psi=0.4 \pi, \tau=1.0$. For such a value of $I_{s}$, the orbital efficiency of the uncoupled 
gyrotrons are about $45 \%$. As predicted by the theory developed in [5], there exist in-phase and anti-phase mode of synchronization.
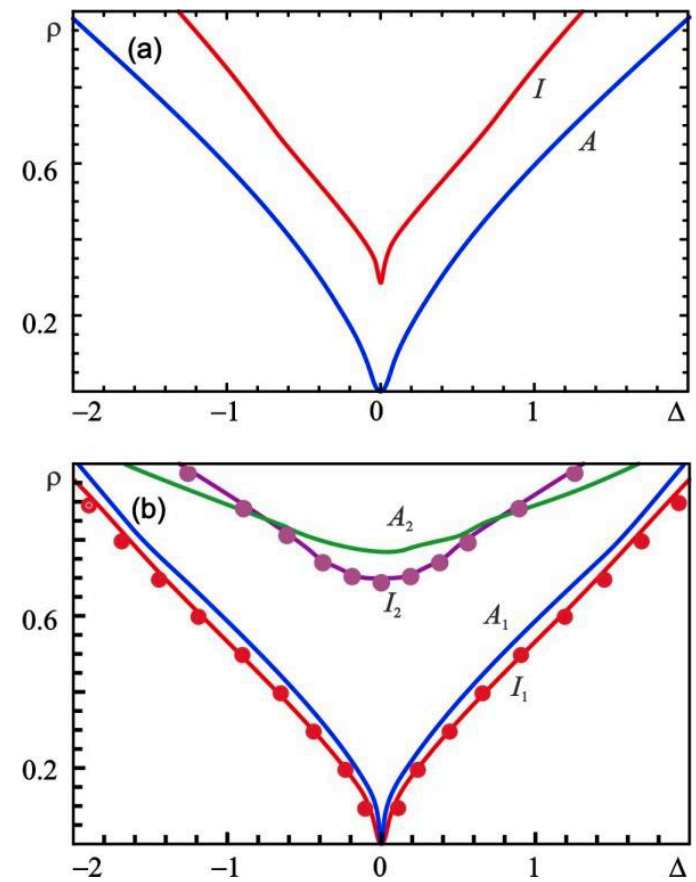

Fig. 2. Domains of stability of the in-phase $(I)$ and anti-phase (A) mode of synchronization on the $\Delta, \rho$ plane for $I_{s}=0.05$, $\psi=0.4 \pi$, and $\tau=1.0$ (a) and 5.0 (b)
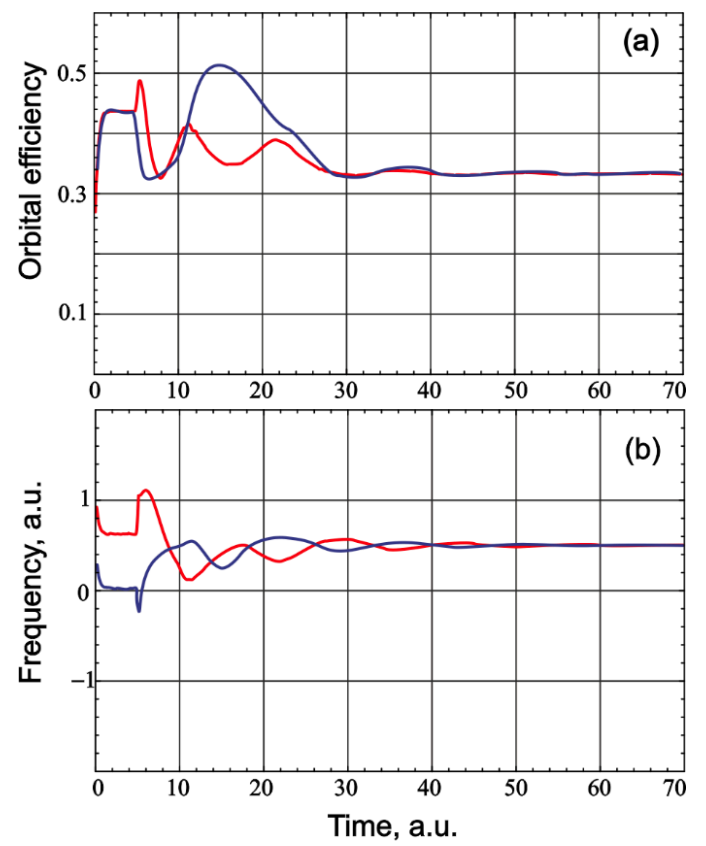

Fig. 3. Time histories of orbital efficiencies (a) and frequencies (b) in the phase-locked mode: $\rho=0.5$, other parameters same as in Fig. 2
With the increase of the delay, there appear new modes of synchronization, i.e. the multistability becomes more pronounced. Fig. 2(b) illustrates the situation when there exist two in-phase and two anti-phase modes.

In addition, in Fig. 2(b) the stability domains calculated according the time-domain gyrotron theory with fixed Gaussian profile of the RF field [7] are shown with circles. They are in good agreement with the results of simulation for the DDE model (1).

In Fig. 3, typical waveforms of orbital efficiencies and instantaneous frequencies of two oscillators are presented. One can see that at the beginning stage of the transient, the gyrotrons oscillate with nearly same efficiencies but with slightly different frequencies. At $t>\tau$ the signal from the first gyrotron begins to affect the second one and vice versa. When the coupling strength is large enough, mutual synchronization takes place. After a rather short transient time the frequencies become equal, which clearly illustrates the effect of frequency locking.

\section{Summary}

Mutual synchronization of two gyrotrons coupled with delay is studied. The model of coupled gyrotrons described by two DDEs (1) is developed. The results of simulation of transition to the mode of synchronization are presented and the conditions of arising of either inphase or anti-phase synchronization are presented. Qualitative agreement with the theory developed in [5] is observed. The results of simulation using the timedomain gyrotron theory with fixed RF field profile are in good agreement with the DDE model.

This work is supported by the Russian Foundation for Basic Research grant No. 16-32-00124.

\section{References}

1. Thumm, M. Recent advances in the worldwide fusion gyrotron development // IEEE Trans. Plasma Sci. 2014. V. 42, No. 3. P. $590-599$.

2. Yakunina, K.A., Kuznetsov, A.P., Ryskin, N.M. Injection locking of an electronic maser in the hard excitation mode // Phys. Plasmas. 2015. V. 22, No 11. 113107.

3. Bakunin, V.L, Denisov, G.G., Novozhilova, Y.V. Zones of frequency locking by an external signal in a multimode gyrotron of a megawatt power level // Radiophys. Quantum Electron. 2016. V. 58, No. 12. P. 893-904.

4. Rozental, R.M., Ginzburg, N.S., Glyavin M.Yu., Sergeev, A.S., Zotova, I.V. Mutual synchronization of weakly coupled gyrotrons // Phys. Plasmas. 2015. V. 22, No. 9. 093118.

5. Usacheva S.A., Ryskin N.M., Phase locking of two limit cycle oscillators with delay coupling // Chaos. 2014. V. 24, No. 2. 023123 .

6. Nusinovich, G. S. Mode interaction in gyrotrons // Int. J. Electron. 1981. V. 51, No. 4. P. 457-474.

7. Nusinovich, G.S. Introduction to the Physics of Gyrotrons. Johns Hopkins University Press, Baltimore, 2004. 